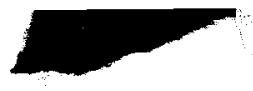

MPERTECTION-SENSTTIVITY OF EXTERNALLY PRESSURIZED ST̈HERICÁL STHELLS

John w. Hutchinson

Harvard University, Cambridge, Massachusetts

\title{
INTRODUCTION
}

In this paper the initial post-buckling behavior of a spherical shell subfect to external pressure loading is determined on the basis of Roiter's general theory of post-buckling behavior. (1) As might well be expected, the most important features of this problem show a striking similarity to aspects present in the behavior of cylindrical shells under axial compression. Imperfections in the sheil geometry are found to have the same severe effect on spherical shells as has been demonstrated for axially compressed cylinders. (1), (2)

Perhaps the main feature which distinguishes this investigation from previous work is that here consideration has not been restricted to rotationallysymmetric buckling deformations. In fect, it is clearly demonstrated that the inltial post-buckling behavior is decidedly not rotationally-symetric but is analogous to the cyilndrical shell behavior in which a number of modes combine to give rise to the highly imperfection-sensitive character of the structure.

Thompson (3) has also employed the Kolter theory to study the Initial postbuckling behavior of the complete sphere. His approach, however, is fundamentally different than that taken here because of the restriction to rotationally-symetric deformations. Th1s work will be discussed further in $\star$

This work was supported in part by the National Aeronautics and Space Administration under Grant NsG-559, and by the Division of Engineering and Applied Physics, Harvard University.
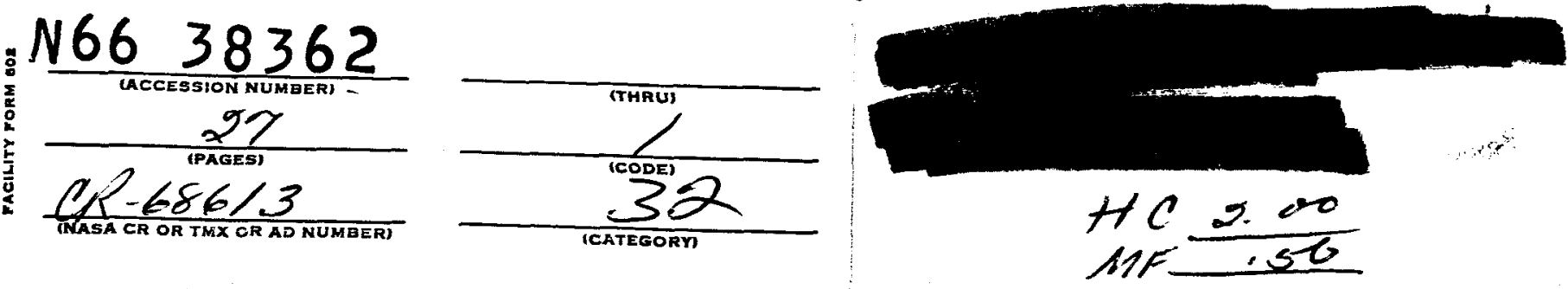
the body of the present paper. Other Investigators $(4),(5),(6)$ have determined the large-deflection behavior with the aid of various methods but in each case under the above mentloned assumption of rotational symetry. The largedeflection, rotationally-symmetric equilibrium configurations appear to be in reasonable agreement with experimental observations in the same large-deflection range.

To obtain a ciear understanding of the effects of imperfections on the buckling strength of this structure it is necessary to study its inftial postbuckling behavior. It is this study which forms the substance of the present paper.

\section{SHALLOW SHELL EQUATIONS}

Nonlinear shallow shell equations will be employed in this analysis. The consistency of applying this representation to the complete sphere will be discussed as the analysis proceeds. In anticipation, however, we remark that the adequacy of this description follows from the fact that the characteristic buckle wave lengths are small compared to the shell radius. Thus, it is possible to choose a shallow section of the shell surface in which the buckle pattern is duplicated many times. ${ }^{\dagger}$ For essentially the same reason, the initial postbuckling behavior of an axially compressed cylinder can also be obtained within the context of shallow shell theory (which for cylinders is Identical to nonIinear Donnell theory).

A shallow section $S_{0}$ of the sphere is imagined to be isolated as shown $+$

Here shallow is taken in the sense that the slopes of the surface measured from the section base are small and, thus, the shallow shell approximations are valid.

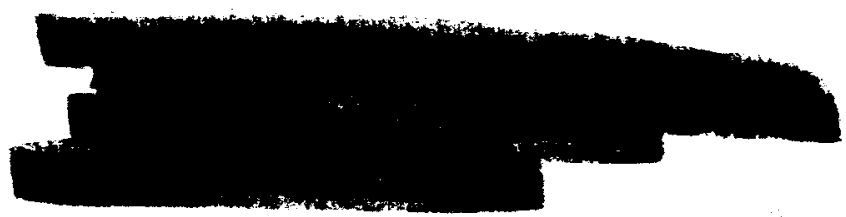


in Figure 1. Cartesian coordinates $x$ and $y$ are chosen in the base plane of the shallow section and $z$ is normal to this plane. The stress-strain relations and the bencing strain-displacement relations of shallow shell theory are linear while the membrane strain-displacement relations are nonlinear. Listed here are these nonlinear relations, which along with the other shallow shell equations, are given, for example, by Sanders. (7) The membrane strains $\varepsilon_{x}=\varepsilon_{y}$ and $\varepsilon_{x y}$ are given in terms of the tangential displacements $U$ and $V$ and the normal displacement $W$ by

$$
\begin{aligned}
& \varepsilon_{x}=U_{, x}+W / R+\frac{1}{2} W_{, x}^{2} \\
& \varepsilon_{y}=V_{, y}+W / R+\frac{1}{2} W_{, y}^{2} \\
& \varepsilon_{x y}=\frac{1}{2}\left(U, y+V_{, x}\right)+\frac{1}{2} W_{, x}, y
\end{aligned}
$$

where $R$ is the radius of curvature of the spherical section.

The three equilibrium equations of nonlinear shallow shell theory can be replaced by one equilibrium equation and one compatibility equation written in terms of $W$ and a stress function $F$. These two equations are

$$
\begin{aligned}
& D \nabla^{4} W+\frac{1}{R} \nabla^{2} F-F, x x^{W}, y y-F, y y, x x+2 F, x y, x y=-p \\
& \frac{1}{E h} \nabla^{4} F-\frac{1}{R} \nabla^{2} W+W, x x, y y-W^{2}, x y=0
\end{aligned}
$$

where $D=E h^{3} / 12\left(1-v^{2}\right), E$ and $v$ are Young's Modulus and Poisson's Ratio, $h$ is the shell thickness, $\nabla^{4}$ and $\nabla^{2}$ are the two-dimensional biharmonic and Laplacian operators, and $p$ is the external pressure. The resultant membrane stresses are given in terms of the stress function by

$$
N_{x}=F_{, y y}, N_{y}=F_{, x x} \text { and } N_{x y}=-F, x y
$$




\section{- CLASSICAL BUCKLING ANALYSIS}

Prior to buckling tie perfect spherical shell is in a uniform membrane state of stiess $\left(N_{x}^{0}=N_{y}^{0}=-\frac{1}{2} p R\right)$ with an associated inward radial displacement $\mathrm{w}^{0}=-(1-v) p R^{2} / \mathrm{Eh}$. With

$$
F=-\frac{1}{4}\left(x^{2}+y^{2}\right) p R+f
$$

and

$$
W=-(1-v) p R^{2} / E h z+w
$$

$f$ and $w$ are zero prior to buckling. The critical pressure $p_{c}$, often called the classical buckling pressure, at which bifurcation from the prebuckling state of stress occurs is predicted by the linear buckling analysis. The linear buckling equations are obtained by substituting for $F$ and $W$ into Equations (2) and (3) and then linearizing with respect to $f$ and $w$. One finds

$$
D \nabla^{4} w+\frac{1}{R} \nabla^{2} f+\frac{1}{2} p R \nabla^{2} w=0
$$

and

$$
\frac{1}{E h} \nabla^{4} f-\frac{1}{R} \nabla^{2} w=0
$$

Periodic solutions to these homogeneous eigenvalue equations are sought in the form of products of sinusoldal functions such as

$$
\begin{aligned}
& w=\cos \left(k_{x} \frac{x}{R}\right) \cos \left(k_{y} \frac{Y}{R}\right) \\
& f=B \cos \left(k_{x} \frac{X}{R}\right) \cos \left(k_{y} \frac{Y}{R}\right)
\end{aligned}
$$

The eigenvalue assoctated with this choice is

$$
p=\frac{2 E h}{R}\left[\left(k_{x}^{2}+k_{y}^{2}\right)^{-1}+q_{0}^{-4}\left(k_{x}^{2}+k_{y}^{2}\right)\right]
$$

with $B=-\operatorname{EhR}\left(k_{x}^{2}+k_{y}^{2}\right)^{-1}$ and where

$$
q_{0}^{4}=12\left(1-v^{2}\right)\left(\frac{R}{h}\right)^{2}
$$


- The classical buckling pressure is found by minimizing $p$ as given by Equation (7) with respect to $k_{x}$ and $k_{y}$. This critical pressure is

$$
p_{c}=4 E h / R q_{0}^{2}=\frac{2 E}{\sqrt{3\left(1-v^{2}\right)}}\left(\frac{h}{R}\right)^{2}
$$

and is associated with any combination of wave numbers $k_{x}$ and $k_{y}$ satisfying

$$
k_{x}^{2}+k_{y}^{2}=q_{0}^{2}
$$

This critical pressure, obtained on the basis of shallow shell theory, is exactly that predicted by equations for a full sphere (see, for example, Flügge ${ }^{(8)}$ ).

The shallow shell representation of the portion $s_{0}$ of the complete sphere can only be valid if the wave lengths of the buckle pattern are small compared to the radius of the shell, or what is the same, if the wave numbers $k_{x}$ and $k_{y}$ are both large compared to unity. Assoclated with the critical buckling pressure is a multiplicity of buckling modes and, as seen from Equation (9), combinations of $k_{x}$ and $k_{y}$ are possible such that both are of order $q_{0}$ and, therefore, sufficiently large. An exception to the requirement of large wave numbers occurs if either $k_{x}$ or $k_{y}$ is identically zero, that 1s, if the buckling deformation is independent of either $x$ or $y$. In such cases, as well, the shallow shell description is accurate for shallow sections of a complete sphere. This 1 s analogous to the situation for axial buckling of cylinders for which the shallow shell equations are accurate for the axisymmetric mode but not, for example, for the Euler column mode in which only one wave length, in effect, spans the shell c1rcumference.

The initial post-buckling behavior of the spherical shell is investigated In the remalnder of this paper. It w111 be seen that sets of efther two or three of the buckling modes associated with the classical buckling pressure 
- couple to give rise to a load-deflection behavior which falls sharply in the initial post-buckling regime. As previously indicated, the analysis will be carried out within the framework of Koiter's general theory of post-buckling behavior. This theory is outlined in the next section.

\section{KOTTER THEORY FOR IMLTI-MODE BUCKLING}

The procedure which is sketched below is an application of the variational principle of potential energy to obtain equations characterizing equilibrium in the prebuckling and inftial post-buckling regimes of a structure with a multiplicity of buckling modes assoclated with the critical buckling load. These equations are in the form of simultaneous nonlinear, algebraic equations relating the magnitude of the externally applied load to the deflections in the various buckling modes. The magnitudes of assumed geometrical imperfections also appear. The notation and development of Koiter's general theory displayed here 18 taken for the most part from Reference 9. The reader is referred to this reference or Koiter's ${ }^{(1)}$ own work for certain arguments and points of rigor which there is no need to re-establish here.

Generalized stress, strain and displacement fieids are denoted by $\sigma$, $\varepsilon$ and $u$, respectively. The magnitude of the applied load system is taken to be directly proportional to the load parameter $\lambda$.

The potential energy expression for the structure is conveniently written In the compact form

$$
P E=\frac{1}{2}\{\sigma, \varepsilon\}-\lambda B_{1}(u)
$$

where, of course, the stresses and strains are calculated from the kinematically admisstble displacement field $u$. Here, in general, $\left[\sigma^{\prime}, \varepsilon^{\prime \prime}\right\}$ denotes the interal virtual work of the stress field $\sigma^{\prime}$ through the strain field $\varepsilon^{\prime \prime}$; 
and $\lambda B_{1}(u)$ is the work of the applied force field of intensity $\lambda$ through a displacement $u$ of the structure.

We consider only structures which can be adequately described by nonlinear strain-displacement relations of the form

$$
\varepsilon=L_{1}(u)+\frac{1}{2} L_{2}(u)
$$

where $L_{1}$ and $L_{2}$ are homogeneous functionals which are linear and quadratic, respectively, in $u$. Furthermore, the stress-strain relations are assumed to be linear and are written symbolically as

$$
\sigma=H_{1}(\varepsilon)
$$

where $H_{1}$ is a linear, homogeneous functional of the strain components. The set of nonlinear shallow shell equations are of this form. With this notation, for example, $L_{2}$ is zero in calculating the bending strain while $L_{2}$ is $w_{, x}^{2}$ in calculating $\epsilon_{x}$.

An Initial deviation $\bar{u}$ of the unloaded structure from the perfect form is called the initial imperfection. In the presence of an initial imperfection the strain arising from an additional displacement $u$ is

$$
\varepsilon=L_{1}(u)+\frac{1}{2} L_{2}(u)+L_{11}(u, \bar{u})
$$

where $i_{11}(u, \bar{u})=L_{11}(\bar{u}, u)$ is the bilinear, homogeneous functional of $u$ and $\bar{u}$ which appears in the Identity

$$
L_{2}(u+\bar{u})=L_{2}(u)+2 L_{11}(u, \bar{u})+L_{2}(\bar{u})
$$

(As an illustration, if the Initial deviation of the shell middle surface from a spherical shape is denoted by $\bar{W}$ then by shallow shell theory $\varepsilon_{x}$ 1s, using (1) and (13), $U, x+W / R+\frac{1}{2} w_{, x}^{2}+V, x^{W}, x$ where $w$ is the additional radial displacement.)

It is assumed that there are several linearly independent buckling modes 
$u_{c}^{(i)}, u_{c}^{(2)}, \ldots$, assoclated with the critical value of the load parameter

$\lambda_{c}$. The complete displacement of the structure is written quite generally as

$$
u=\lambda u_{0}+\sum_{n} \xi_{n} u_{c}^{(n)}+\tilde{u}
$$

where $\lambda u_{0}$ is the prebuckling displacement of the perfect structure subject to the external load intensity corresponding to $\lambda$. For the spherical shell under uniform pressure this is just a uniform radial displacement. Each of the modes $u_{c}^{(1)}$ are taken orthogonal to one another and each is orthogonal to $\mathfrak{u}$. The orthogonality condition is

$$
\left\{\sigma_{0}, L_{11}\left(u_{c}^{(i)}, u_{c}^{(j)}\right)\right\}=0 \quad 1 \neq j
$$

where $\sigma_{0}=H_{1}\left[L_{1}\left(u_{0}\right)\right]$. Imperfections in the form of the buckling modes/result in significant reductions of the buckling load; thus, we take

$$
\overline{\mathbf{u}}=\sum_{\mathbf{n}} \bar{\xi}_{\mathbf{n}} \mathbf{u}_{c}^{(\mathbf{n})}
$$

Now, the potential energy is evaluated using the expression for $u$ and $\bar{u}$ with Equations (12)-(14) and the orthogonality conditions. The result is

$$
\begin{aligned}
P E=\text { const. } & +\frac{1}{2}\left(\lambda-\lambda_{c}\right)\left\{\xi_{i}^{2}\left\{\sigma_{0}, L_{2}\left(u_{c}^{(i)}\right)\right\}\right. \\
& +\frac{1}{2}\left\{\sum \xi_{1} s_{c}^{(i)}, L_{2}\left(\left[\xi_{1} u_{c}^{(i)}\right)\right\}+\left[\xi_{1} \bar{\xi}_{1} \lambda\left\{\sigma_{0}, L_{2}\left(u_{c}^{(i)}\right)\right\}\right.\right. \\
& + \text { terms of order } \xi^{4}, \xi \bar{\xi}^{2}, \ldots
\end{aligned}
$$

where $s_{c}^{(1)}=H_{1}\left[L_{l}\left(u_{c}^{(1)}\right)\right]$. Only terms up to and Including third powers of the $\xi^{\prime} s$ and imperfection terms like $\bar{\xi}$ in the potential energy are displayed. This expression for a "quadratic structure" is in the form given by Kolter [(1), Eq. (28)]. It is noted that $\tilde{u}$ does not appear In potential energy since it contributes to quartic but not cubic terms in the $\xi^{\prime} s$. The potential 
energy expression in the truncated form given here can provide an accurate description of the structure only so long as the $\xi^{\prime} s$ and imperfections $\bar{\xi}^{\prime} s$ are sufficiently small to insure that the terms neglected are small compared to those retained.

Equilibrium equations relating the $\xi_{1}$ 's to the load parameter $\lambda$ are obtained from the requirement that the first variations of the potential energy with respect to the $\xi_{1}^{\prime}$ 's vanish. These equations are

$$
\begin{aligned}
\xi_{i}\left(1-\lambda / \lambda_{c}\right) & +\left[\left\{\left[\xi_{I} s_{c}^{(n)}, L_{11}\left(\sum_{n_{n}} u_{c}^{(n)}, u_{c}^{(1)}\right)\right\}\right.\right. \\
& +\frac{1}{2}\left\{s_{c}^{(i)}, L_{2}\left(\left[\xi_{n} u_{c}^{(n)}\right)\right\}\right] /\left(-\lambda_{c}\left\{\sigma_{o}, L_{2}\left(u_{c}^{(1)}\right)\right\}\right) \\
& =\frac{\lambda}{\lambda_{c}} \bar{\xi}_{1} \quad 1=1,2, \ldots
\end{aligned}
$$

fin.ally, we give the generalized load-deflection relation for a perfect, multi-mode structure, obtained from Equation (10) with the aid of the auxiliary equations, namely

$$
\frac{B_{1}(u)}{B_{1}\left(u_{o c}\right)}=\frac{\lambda}{\lambda_{c}}-\frac{1}{2} \sum_{n} \xi_{n}^{2} \frac{\lambda_{c}\left\{\sigma_{o}, L_{2}\left(u_{c}^{(n)}\right)\right\}}{\lambda_{c}^{2}\left\{\sigma_{o}, L_{1}\left(u_{o}\right)\right\}}
$$

where $u_{o c}=\lambda_{c} u_{0}$ is the prebuckling displacement at the buckling load and from Equation $(10), B_{1}(u)$ represents the generalized displacement through which the external loading system acts.

FUSI-BUCKLING UF SPHERICAL SHELL: TWO OPERATIVE MODES

In forming the nonlinear equations of equilibrium for the spherical shell It is necessary to take into account all the buckling modes associated with the critical buckling pressure, that is, all modes whose wave numbers satisfy

$$
k_{x}^{2}+k_{y}^{2}=q_{0}^{2}
$$

It will be shown that the nonlinear equations for the $\xi^{\prime}$ 's decouple into separate sets of equations corresponding to interaction between either two or 
three of these critical modes. Two modes will interact when one of the operative modes has a zero wave number associated with either the $x$ or $y$ coordinate. This case is considered first and the three mode situation is alscussed in the next section.

Translating frow the general notation to the shallow shell notation, we take as $u_{c}^{(1)}$

$$
w_{c}^{(1)}=h \cos \left(q_{0} \frac{x}{R}\right)
$$

with the associated stress function

$$
f_{c}^{(1)}=-E R h^{2} q_{o}^{-2} \cos \left(q_{0} \frac{x}{R}\right)
$$

of all the modes satisfying (9), only a mode with $k_{x}=q_{0} / 2$, and thus $k_{y}=\sqrt{3} q_{o} / 2$, will interact with $w_{c}^{(1)}$, as will be shown. For $u_{c}^{(2)}$ take

$$
\begin{gathered}
w_{c}^{(2)}=h \sin \left(\frac{1}{2} q_{0} \frac{x}{R}\right) \sin \left(\frac{\sqrt{3}}{2} q_{0} \frac{y}{R}\right) \\
f_{C}^{(2)}=-E R h^{2} q_{O}^{-2} \sin \left(\frac{1}{2} q_{0} \frac{x}{R}\right) \sin \left(\frac{\sqrt{3}}{2} q_{0} \frac{y}{R}\right)
\end{gathered}
$$

The coefficients of the various terms in the equilibrium equations (17) are easily calculated. Some of the details are shown below. First

$$
\begin{aligned}
\lambda_{c}\left\{\sigma_{0}, L_{2}\left(u_{c}^{(1)}\right)\right\} & =-\frac{1}{2} p_{c}^{R} \int_{S_{0}}\left(w_{c, x}^{(1)}\right)^{2} d s \\
& =-\frac{2 E h^{3}}{R^{2}} \int_{S_{0}}\left(\sin q_{0} \frac{x}{R}\right)^{2} d s \\
& =-\frac{E h^{3}}{R^{2}} s_{0}
\end{aligned}
$$


where $S_{0}$ is the area of the shaliow section. Consistent with the fact that the buckle wave length is short compared to the characteristic length of the section $S_{0}$ only the constant part of $\sin ^{2} q_{0} \frac{x}{R}=\frac{1}{2}\left(1-\cos 2 q_{0} \frac{x}{R}\right)$ is evaluated in arriving at the above expression. Similarly

$$
\lambda_{c}\left\{\sigma_{o}, L_{2}\left(u_{c}^{(2)}\right)\right\}=-\frac{E h^{3}}{2 R^{2}} s_{o}
$$

and

$$
\begin{aligned}
\left\{s_{c}^{(1)}, L_{2}\left(u_{c}^{(2)}\right)\right\} & =\int_{S_{0}} f_{c, x x}^{(1)}\left(w_{c, y}^{(2)}\right)^{2} d S \\
& =-\frac{3 C}{16} \frac{E h^{3}}{R^{2}} s_{0}
\end{aligned}
$$

where $C=\sqrt{3\left(1-v^{2}\right)}$. This last coefficient, which gives rise to coupling between $w_{c}^{(1)}$ and $w_{c}^{(2)}$, would vanish if the $k_{x}$ associated with $w_{c}^{(2)}$ were not $\frac{1}{2} q_{0}$. The other non-zero coupling coefficient is

$$
\begin{aligned}
\left\{s(2), L_{1}\left(u_{c}^{(1)}, u_{c}^{(2)}\right)\right\} & =\int_{S}\left[f_{c, y y}^{(2)} w_{c, x}^{(1)} w_{c, x}^{(2)}-f_{c, x y}^{(2)} w_{c, x}^{(1)} w_{c, y}^{(2)}\right] d S \\
& =-\frac{3 C}{16} \frac{E h^{3}}{R^{2}} s_{0}
\end{aligned}
$$

while

$$
\begin{aligned}
\left\{s_{c}^{(1)}, L_{11}\left(u_{c}^{(1)}, u_{c}^{(2)}\right)\right\} & =\left\{s_{c}^{(1)}, L_{2}\left(u_{c}^{(1)}\right)\right\}=\left\{s_{c}^{(2)}, L_{2}\left(u_{c}^{(2)}\right)\right\} \\
& =\left\{s_{c}^{(2)}, L_{2}\left(u_{c}^{(1)}\right)\right\}=0
\end{aligned}
$$

Last1y,

$$
\lambda_{c}^{2}\left\{\sigma_{0}, L_{1}\left(u_{0}\right)=\frac{2}{3(1+v)} \frac{E h^{3}}{R^{2}} s_{0}\right.
$$

With these coefficients the two equilibrium equations for $\xi_{1}$ and $\xi_{2}$ in terms of the external pressure $p$ become

$$
\left(1-\frac{p}{p_{c}}\right) \xi_{1}-\frac{9 C}{32} \xi_{2}^{2}=\frac{p}{p_{c}} \bar{\xi}_{1}
$$




$$
\left(1-\frac{p}{p_{c}}\right) \xi_{2}-\frac{9 C}{8} \xi_{1} \xi_{2}=\frac{p}{p_{c}} \bar{\xi}_{2}
$$

and it is Important to note that the deflections in the modes, $\xi_{1}$ and $\xi_{2}$, as well as the Imperfection magnitudes, $\bar{\xi}_{1}$ and $\bar{\xi}_{2}$, are measured relative to the shell thickness. This follows from the choice of $w_{c}^{(1)}$ and $w_{c}^{(2)}$ in Equations (19) and (20). $\dot{\top}$

For the perfect she11 $\left(\bar{\xi}_{1}=\bar{\xi}_{2}=0\right)$ Equations (21) and (22) admit only the trivial solution when the prebuckling pressure is less than the critical value $P_{c}$. When $p$ attains $P_{c}$ bifurcation from the membrane state of stress occurs, and the equilibrium equations are easily solved for $\xi_{1}$ and $\xi_{2}$ :

$$
\begin{aligned}
& \xi_{1}=\frac{3}{9 C}\left(1-\frac{p}{p_{c}}\right) \\
& \xi_{2}= \pm \frac{16}{9 C}\left(1-\frac{p}{p_{c}}\right)
\end{aligned}
$$

Th1s behavior, sketched in FIgure 2, is characteristic of a "quadratic type" structure and has been discussed by Koiter ${ }^{(1)}$ in some detail for the general case. The equilibrium pressure in the post-buckling regime is greatly reduced even where the buckling deflections are only a small fraction of the shell thickness, i.e., $\xi_{1}$ and $\xi_{2}$ a small fraction of unity.

The generalized load-deflection relation valid in the fnitial post-buckling region is easily calculated using Equation (18). The generalized displacement $B_{1}(u)$ In this case corresponds to the average normal displacement, wave, of the shallow section. One finds

$$
\frac{w_{\text {ave }}}{w_{c}^{0}}=\frac{p}{P_{c}}+\frac{16}{27(1-v)}\left(1-\frac{p}{P_{c}}\right)^{2}
$$

where $W_{c}^{0}=-\sqrt{\frac{1-v}{3(1+v)}}$ h is the prebuckling normal displacement at the Note that Equations (21) and (22) as well as the predictions to be obtained from them do not depend on the area of the shallow section $s_{0}$. 
- bifurcation pressure.

An Imperfect shell suffers deflections in the buckling modes with the first application of external pressure. The behavior for the case $\bar{\xi}_{2}=0$, $\bar{\xi}_{1}>0$ is also depicted in Figure 2. Prior to buckling the load Increases with deflection in the $\xi_{1}$ mode with

$$
\xi_{1}=\frac{\bar{\xi}_{1} p / p_{c}}{1-p / p_{c}}
$$

unt1l the coefficient of $\xi_{2}$ in Equation (22) vanishes. At this point bifurcation occurs. Following bifurcation the equilibrium pressure falls with deflections occurring in both modes; and thus, the maximum (buckling) pressure, denoted by $p^{*}$, is the bifurcation pressure which satisfies

$$
\left(1-\frac{p^{*}}{p_{c}}\right)^{2}=\frac{9 c}{8} \bar{\xi}_{1} \frac{p^{*}}{p_{c}}+
$$

Small Imperfections (relative to the shell thickness) result in large reductions of the buckling pressure as shown in the plot of (25) in Figure 3.

If $\bar{\xi}_{1}=0$ but $\bar{\xi}_{2} \neq 0$, the maximum value of $p$ is obtained by substituting for $\xi_{1}$ in terms of $\xi_{2}$ from Equation (21) into Equation (22) and determining then the value of $p$ such that $\frac{d p}{d \xi_{2}}=0$. One finds

$$
\left(1-\frac{\mathrm{p}^{*}}{\mathrm{P}_{\mathrm{c}}}\right)^{2}=\frac{27 \sqrt{3} \mathrm{C}}{32}\left|\bar{\xi}_{2}\right| \frac{\mathrm{p}^{*}}{\mathrm{P}_{\mathrm{c}}}
$$

This formula is also plotted in Figure 3 and it is seen that an imperfection In the form of the $\xi_{2}$ mode causes a.greater reduction than an equal imperfection In the $\xi_{1}$ node.

\section{THREE OPERATIVE BUCKLING MODES}

Interaction between two modes occurs only if one mode has a zero wave

$t$ If we had chosen $w_{c}^{(2)}=h \cos \left(q_{0} x / 2 R\right) \sin \left(\sqrt{3} q_{0} y / 2 R\right)$ Instead of (20), Equation (25) would be $\left(1-p^{*} / p_{c}\right)^{2}=-9 c \bar{\xi}_{1 p}{ }^{*} / 8 p_{c}$ and thus valld for $\bar{\xi}_{1}<0$. 
number; otherwise, the modes will interact in sets of three. To 11lustrate such situation we take as $u_{c}^{(1)}, u_{c}^{(2)}$ and $u_{c}^{(3)}$

$$
\begin{aligned}
& {\left[\begin{array}{l}
w_{c}^{(1)} \\
f_{c}^{(1)}
\end{array}\right]=\left[\begin{array}{c}
h \\
-E R h^{2} q_{0}^{-2}
\end{array}\right] \cos \left(\alpha_{1} q_{0} \frac{x}{R}\right) \cos \left(\beta_{1} q_{0} \frac{y}{R}\right)} \\
& {\left[\begin{array}{l}
w_{c}^{(2)} \\
f_{c}^{(2)}
\end{array}\right]=\left[\begin{array}{c}
h \\
-E R h^{2} q_{0}^{-2}
\end{array}\right] \sin \left(\alpha_{2} q_{0} \frac{x}{R}\right) \sin \left(\beta_{2} q_{0} \frac{z}{R}\right)} \\
& {\left[\begin{array}{l}
w_{c}^{(3)} \\
f_{c}^{(3)}
\end{array}\right]=\left[\begin{array}{c}
h \\
-E R h^{2} q_{0}^{-2}
\end{array}\right] \sin \left(\alpha_{3} q_{0} \frac{x}{R}\right) \sin \left(\beta_{3} q_{0} \frac{q}{R}\right)}
\end{aligned}
$$

and

where by, Equation (9), $a_{1}^{2}+\beta_{1}^{2}=1(1=1,2,3)$.

The coefficients in the algebraic equilibrium equations are evaluated in much the same manner as in the last section. Coupling between the three modes w111 occur only if coefficients such as $\left\{s_{c}^{(1)}, \mathrm{L}_{11}\left(u_{c}(2), u_{c}^{(3)}\right)\right\}$ do not vanish. Evaluating this term we find

$$
\begin{aligned}
& \left\{s_{c}^{(1)}, L_{11}\left(u_{c}^{(2)}, u_{c}^{(3)}\right)\right\}=\int_{S_{0}}\left\{f_{c, y y}^{(1)} w_{c, x}^{(2)_{w}} w_{c, x}^{(3)}+f_{c, x x}^{(1)} w_{c, y}^{(2)_{w}(3)}\right. \\
& \left.-f_{c, x y}^{(1)}\left(w_{c, x}(2)_{c, y}(3)_{c, y}+w_{c, x}^{(2)_{w}(3)}\right)\right] d S \\
& =-\frac{C}{8} \frac{E h^{3}}{R^{2}} S_{0}\left(-\beta_{1}^{2} \alpha_{2} \alpha_{3}+\alpha_{1}^{2} \beta_{2} \beta_{3}-\alpha_{1} \beta_{1} \alpha_{3} \beta_{2}+\alpha_{1} \beta_{1} \alpha_{2} \beta_{3}\right)
\end{aligned}
$$

if $\alpha_{2}+\alpha_{3}=\alpha_{1}$ and $B_{1}+B_{2}=B_{3}$ but is zero otherwise. ${ }^{t}$ These two equations along with the conditions $\alpha_{1}^{2}+\beta_{1}^{2}=1$ uniquely determine the magnitudes of any five of the $\alpha^{\prime} s$ and $\beta^{\prime}$ 's in terms of the remalning one. From this follows the

Actually there are other combinations for which this coefficient will not vanish, for example $\alpha_{2}+\alpha_{3}=-\alpha_{1}$ and $\beta_{1}+\beta_{2}=-\beta_{3}$, but these are, in effect, included with the combination ilsted above. 
' statement made eariler that the equilibrium equations decouple into sets made up of three interacting modes unless, of course, one mode wave number is zero. Then the three mode case degenerates to the case discussed in the last section.

The remaining nonlinear, coupling coefficlents are non-vanishing only under the same conditions and are easily found to be

and

$$
\left\{s_{c}^{(2)}, L_{11}\left(u_{c}^{(1)}, u_{c}^{(3)}\right)\right\}=-\frac{C}{8} \frac{E h^{3}}{R^{2}} s_{0}\left(\beta_{2}^{2} \alpha_{1} \alpha_{3}+a_{2}^{2} \beta_{1} \beta_{3}+\alpha_{2} \beta_{2} \alpha_{1} \beta_{3}+\alpha_{2} \beta_{2} \beta_{1} \alpha_{3}\right)
$$

$$
\left\{s_{c}^{(3)}, L_{11}\left(u_{c}^{(1)}, u_{c}^{(2)}\right)\right\}=-\frac{C}{8} \frac{E h^{3}}{R^{2}} s_{0}\left(\beta_{3}^{2} \alpha_{1} \alpha_{2}-\alpha_{3}^{2} \beta_{1} \beta_{2}+\alpha_{3} \beta_{3} \alpha_{1} \beta_{2}-\alpha_{3} \beta_{3} \beta_{1} \alpha_{2}\right)
$$

wth all others zero. Finally,

$$
\lambda_{c}\left\{\sigma_{o}, L_{2}\left(u_{c}^{(1)}\right)\right\}=-\frac{1}{2} \frac{E h^{3}}{R^{2}} s_{0} \quad I=1,2,3
$$

The coefficient of the nonlinear term in each of the three equilibrium equations $18\left\{s_{c}^{(1)}, L_{11}\left(u_{c}^{(2)}, u_{c}^{(3)}\right)\right\}+\left\{s_{c}^{(2)}, L_{11}\left(u_{c}^{(1)}, u_{c}^{(3)}\right)\right\}+\left\{s_{c}^{(3)}, L_{11}\left(u_{c}^{(1)}, u_{c}^{(2)}\right)\right\}$ This coefficient reduces to a constant value, $-\frac{9 \mathrm{C}}{32} \frac{\mathrm{Eh}^{3}}{\mathrm{R}^{2}} \mathrm{~s}_{0}$, independent of which three mode set is under consideration. Thus, the three equilibrium equations for a given decoupled set are

$$
\begin{aligned}
& \left(1-\frac{p}{p_{c}}\right) \xi_{1}-\frac{9 C}{16} \xi_{2} \xi_{3}=\frac{p}{p_{c}} \quad \bar{\xi}_{1} \\
& \left(1-\frac{\mathrm{p}}{\mathrm{P}_{\mathrm{C}}}\right) \xi_{2}-\frac{9 \mathrm{C}}{16} \xi_{1} \xi_{3}=\frac{\mathrm{P}}{\mathrm{P}_{\mathrm{C}}} \bar{\xi}_{2} \\
& \left(1-\frac{\mathbf{p}}{\mathbf{P}_{\mathrm{C}}}\right) \xi_{3}-\frac{9 \mathrm{C}}{16} \xi_{1} \xi_{2}=\frac{\mathrm{p}}{\mathbf{P}_{\mathrm{C}}} \bar{\xi}_{3}
\end{aligned}
$$

If a three mode set is operative in the initial post-bukcling deformation of the perfect sphere, then

$$
\xi_{1}=\xi_{2}=\xi_{3}=\frac{16}{9 \mathrm{C}}\left(1-\frac{\mathrm{p}}{\mathrm{p}_{\mathrm{c}}}\right)
$$


- and the generalized load-deflection relation, again using Equation (18), Is

$$
\frac{w_{\text {ave }}}{w_{c}^{0}}=\frac{p}{P_{c}}+\frac{.32}{27(1-\nu)}\left(1-\frac{p}{p_{c}}\right)^{2}
$$

One notes that the load-deflection relation for the three mode case differs from that for the two mode situation. The initial post-buckling load-deflection curves for both cases are plotted in Figure 4.

If the shell is imperfect with an imperfection in on1y one of the modes, $\bar{\xi}_{3}>0$ say, the prebuckling deformation is in the $\xi_{3}$ mode

$$
\xi_{3}=\frac{\bar{\xi}_{3} p / p_{c}}{1-p / p_{c}}
$$

Bifurcation from this solution will occur at the value of $p$ when the determinant of the coefficients of $\xi_{1}$ and $\xi_{2}$ in Equations (28) and (29) vanishes. This gives the buckling pressure of the imperfect shell which $1 \mathrm{~s}$ found to be

$$
\left(1-\frac{p^{*}}{P_{c}}\right)^{2}=\frac{9 C}{16} \bar{\xi}_{3} \frac{p^{*}}{P_{c}}
$$

This relation is plotted, along with the results for two mode situation, in Figure 3. A single imperfection in the three mode case is not as degrading as a single imperfection when two modes are operative.

\section{DISCUSSION OF THE RESULTS}

The equilibrium equations of the general theory decouple into sets of either two or three Interacting modes. The set, or combination of sets, which is actually operative in the initlal post-buckling regime of the perfect sphere Is Indeterminate within the context of the general theory. This indeterminacy parallels the initial post-buckling situation for axlally compressed cylindrical shells which Koiter studied via his general theory. Like the 
- spherical shell, the cylindrical shell has a multiplicity of buckling modes associated with the classical buckling load; but unlike the sphere, all the modes can couple through the nonlinear terms in the equilibrium equations. The relative magnitudes of these modes, however, remain undetermined by the general theory. On the other hand, the load-end shortening relation is uniquely determined by the general theory. The generalized load-deflection relation (1.e., p vs. "ave) for the sphere is not uniquely determined by the general theory but depends on whether a two or three mode set is operative. Quite likely the indeterminacy in both problems would be removed if higher order terms were retained in the potential energy expression.

An imperfection in the form of any given mode has the effect of determining which set of modes will be operative. The imperfection which appears to cause the greatest reduction in the maximum support pressure is that in the form of the $\xi_{2}$ mode of the two mode case (see Equation (26)). For axially compressed cylindrical shells, imperfections in the form of the axisymmetric buckilng mode are most critical. (2) The relation of buckling load to imperfection for cylinders with this imperfection is

$$
\left(1-\frac{\lambda^{*}}{\lambda_{c}}\right)^{2}=\frac{3 C}{2} \bar{\xi} \frac{\lambda^{*}}{\lambda_{c}}
$$

where $\lambda^{*} / \lambda_{c}$ is the ratio of the buckling load to the classical buckling load. Here, the Imperfection magnitude is measured relative to the shell thickness in the same manner as in the analogous formulae in this paper. The effect of the most critical imperfection on each structure is almost identical (compare this equation with Equation (26)).

With the restriction that the deformations be rotationally-symetric, Thompson (3) has shown, on the basis of Kolter's general theory that the slope 
of the pressure-deflection curve is negative in the Initial post-buckling regime. The value of this olope, however, is extremely small implying that although the sphere w111 be imperfection-sensitive it will not necessarily be significantiy 80 . In fact, the rotationally-symmetric analysis indicates that for comparable reductions in theibuckling pressure the imperfection magnitudes must be (relative to the shell thickness) on the order of $\sqrt{\mathrm{R} / \mathrm{h}}$ times the values predicted by the present analysis. The rotational-symetry restriction precludes the possibility of the strong coupling between critical modes which has been demonstrated here.

\section{LIMITATIONS OF THE GENERAL THEORY}

As previously emphasized, one can have confidence in the general theory only when the terms dropped from the potential energy expression are sufficiently small compared to the terms retained. In particular, the larger the imperfections the more one should question the buckling load predictions.

When the Imperfection is in the form of a buckling mode with one zero wave number it is possible to obtain an independent estimate of the buckling pressure of the spherical shell. If we take an imperfection in the form of

$$
\bar{w}=\bar{\xi} h \cos q_{0} \frac{x}{R}
$$

the nonlinear shallow shell equations for an Initially imperfect spherical shell admit an exact solution for the prebuckling deformation of the shell, and the deformation is independent of the $y$ coordinate. At a certain value of the external pressure bifurcation from this $y$-independent deformation occurs. The approximate calculation for determining the relation of the bifurcation pressure to the imperfection is sketched briefly in the Appendix. The analogous calculation for the effect of axisymmetric imperfections on the buckling load 
- of cylindrical shells has been given a careful treatment by Kolter. (2) The method of calculation insures that the estimate of the bifurcation pressure, although approximate, is an upper bound to the actual bifurcation pressure. The results of this calculation are shown in Figure 5 where the upper bound results can be compared with the general theory predictions for the same Imperfection, Equation (25). The agreement, as in the case for the cylindrical shell, is surprisingly good even for Imperfections which reduce the buckling load to as little as thirty percent of the classical value. For small Imperfections the upper bound estimate and the general theory prediction approach each other asymptotically.

\section{APPLICATION TO SPHERICAL CAPS}

The conclusions reached with regard to the effects of imperfections on shallow sections of complete spherical shells obviously apply to spherical caps if the buckling wave lengths are omall compared to the base dimension of the cap. The shortest buckle wave length is

$$
2 \pi\left(12\left(1-v^{2}\right)\right)^{-\frac{1}{4}} \sqrt{\mathrm{Rh}}
$$

To obtain a rough estimate of range in which the results of the present analysis should be at least partially applicable, we will demand, quite arbitrarily, that the above wave length be 1 less than one third the base diameter of the cap. With this constraint it is easily shown that the shell rise 1 to the thickness $h$ must sat1sfy

$$
\sqrt{\frac{H}{h}}>\frac{3 \pi}{2}\left(3\left(1-v^{2}\right)\right)^{-\frac{1}{4}}
$$

In terms of the frequently defined shallowness parameter $\lambda=2\left(3\left(1-v^{2}\right)\right)^{1 / 4} \sqrt{\mathrm{H} / \mathrm{h}}$ this implies that $\lambda>3 \pi$.

It is interesting to note that Huang ${ }^{(10)}$ has calculated the buckling 
presgure for inftially perfect spherfial caps which are clamped on the base edge. For sufficiently large rise to beight, $\lambda>3 \pi$ say, the buckling pressure Is about eighty-five percent of the pressure necessary to buckle a complete perfect sphere with the same radius of curvature and thickness. Bxperimental data in this range shows considerable scatter with buciking pressures in many Instances less than thirty percent of the value predicted for the perfect cap. It seems clear that initial imperfections account for the discrepancy between the experimental data and the results for the initially perfect cap.

\section{APPENDIX: UPPER BOUND CALCULATION}

We consider a shallow spherical section with an Imperfection in the form

$$
\bar{w}=\bar{\xi} h \cos q_{0} \frac{x}{R}
$$

The nonlinear shallow shell equations for an initially imperfect spherical shell admit a relatively simple, exact prebuckling solution for an imperfection of the form $(A-1)$. $^{+}$This solution is

$$
\begin{gathered}
W=\left\{-(1-v) p R^{2} / E h 2+\bar{\xi} h \frac{p / p_{c}}{1-p / p_{c}} \cos q_{0} \frac{x}{R}\right\}+w \\
F=\left\{-\frac{1}{4}\left(x^{2}+y^{2}\right) p R-E h R q_{0}^{-2} \bar{\xi} h \frac{p / p_{c}}{1-p / p_{c}} \cos q_{0} \frac{x}{R}\right\}+f
\end{gathered}
$$

where $w$ and $f$ are zero prior to buckling. Buckling occurs with bifurcation from this $y$-independent prebuckling solution. Thus we look for the value of the pressure $p$ at which the nonlinear shallow shell equatione admit nonzero

+ For a $y$-independent initial imperfection the shallow shell equations are altered by appending the term $-F, y y^{\bar{w}}, x x$ to the left hand side of Equation (2) and $+W_{, y y^{\bar{w}}, x x}$ to the left hand side of Equation (3). 
- solutions for $w$ and $f$. Substituting (A-2) and (A-3) into the full nonlinear equations for an initially imperfect spherical shell and then linearizing with respect to $w$ and $f$, we obtain two homogeneous, nonconstantcoefficient equations in $w$ and $f$ for determining the elgenvalue $p^{*}$. In the interests of brevity these equations will not be 11sted here. The approximate method of solution of this elgenvalue problem, only described below, is the subject of a paper by Koiter ${ }^{(2)}$ for the analogous problem of a cylinder with axisymetric imperfections.

One of the two Iinear elgenvalue equations is a compatibility equation which 18 solved exactly for $f$ in terms of an assumed w

$$
w=\sin \left(\frac{1}{2} q_{0} \frac{x}{R}\right) \sin \left(\gamma q_{0} \frac{Y}{R}\right)
$$

where $\gamma$ is a free parameter. Then, $f$ and $w$ are used in confunction with the Raleigh-Ritz method to solve the second equation, an equilibrium equation, approximately and to obtain an equation for the elgenvalue. The elgenvalue equation relating $p^{*}$ and $\bar{\xi}$ is

$$
\begin{aligned}
\left(1-p^{*} / p_{c}\right)^{2}\left(Q^{2}+1-2 Q p^{*} / p_{c}\right) & -c \gamma^{2} \bar{\xi}\left(1-p^{*} / p_{c}\right)\left(p^{*} / p_{c}+2 / Q\right) \\
& +\left(c \gamma^{2} \bar{\xi}\right)^{2}\left(Q^{-2}+\left(9 / 4+\gamma^{2}\right)^{-2}\right)=0
\end{aligned}
$$

where $Q=1 / 4+\gamma^{2}$.

This approximate method of solution insures that the estimate of the buckling pressure $p^{*}$ for a given imperfection. $\bar{\xi}$ is an upper bound to the exact bifurcation pressure. In calculating $P^{*}$ for a given value of $\bar{\xi}$ the free parameter $Y$ is chosen so that the prediction of $p^{*}$. based on $(A-4)$ is a minimum. The results of these calculations are plotted in Figure 5 where they can be compared with the predictions of the general theory. 
- REFERENCES

1. Koiter, W. T., "Elastic Stability and Post-Buckling Behavior", Proc. Symp. 'Nonlinear Problems', edited by R. E. Langer, University of Wisconsin Press, p. 257 (1963).

2. Koiter, ฟ. T., "The Effect of Axisymmetric Imperfections on the Buckling of Cylindrical She1ls under Axial Compression", Koninkl. Ned. Akad. Wetenschap. Proc. Ser. B, 66, pp. 265-279 (1963).

3. Thompson, J. M. T., "The Rotationally-Symmetric Brancing Behaviour of a Complete Spherical She11", Konink1. Neder1. Akad. Van Wetenschap. Proc. Ser. B, 67, pp. 295-311 (1964).

4. Thompson, J. M. T., "The Elastic Instability of a Complete Spherical Shell", Aero. Quart., XIII, 189 (1962).

5. Gabril'Lants, A. G. and Feodos'ev, V. I., "Axially Symetric Forms of Equilibrium of an Elastic Spherical Shell under Uniformly Distributed Pressure", Prik1. Mat. Mek., 25, 1629 (1961).

6. Sab1r, A. B., "Stress Distribution and Elastic Instability of Spherical She1ls", dissertation University College, Cardiff (1962).

7. Sanders, J. L., "Nonlinear Theories for Thin Shells", Quart. Appl. Math., Vo1. XXI, No. 1, Apri1 (1963).

8. Flügge, W., "Stresses in Shells", Springer-Verlag (1960).

9. Budiansky, B. and Hutchinson, J., "Dynamic Buckling of Imperfection-Sensitive Structures", Proc. of the Eleventh International Congress of Applied Mechanics (1964). (Springer: Julius Spring-Verlag, Berlin, to be published.)

10. Huang, N. C., "Unsymmetrical Buckling of Thin Shallow Spherical Shells", J. App1. Mech., September (1964). 


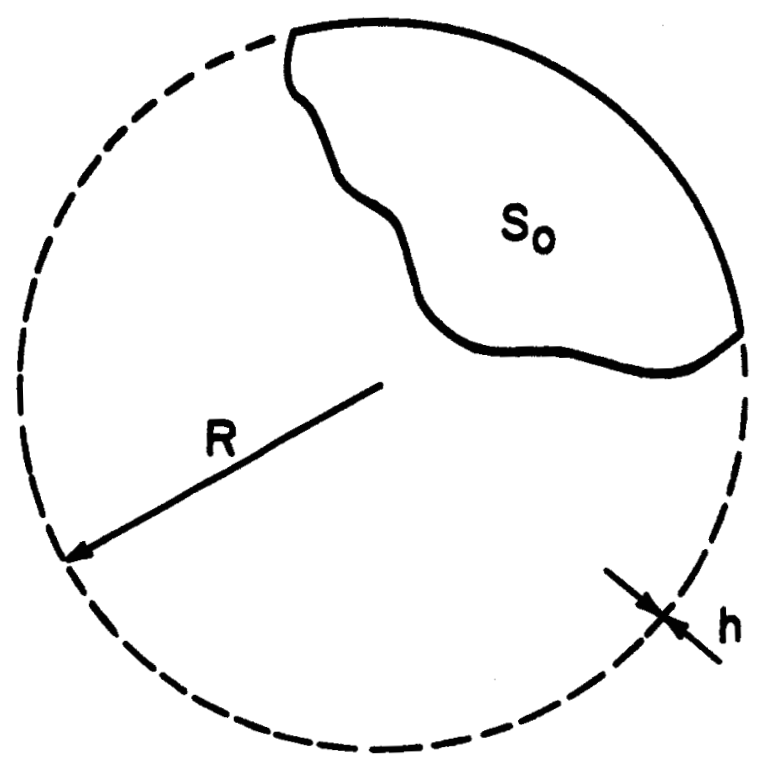

FIG. 1 SHALLOW SECTION OF COMPLETE SPHERE 


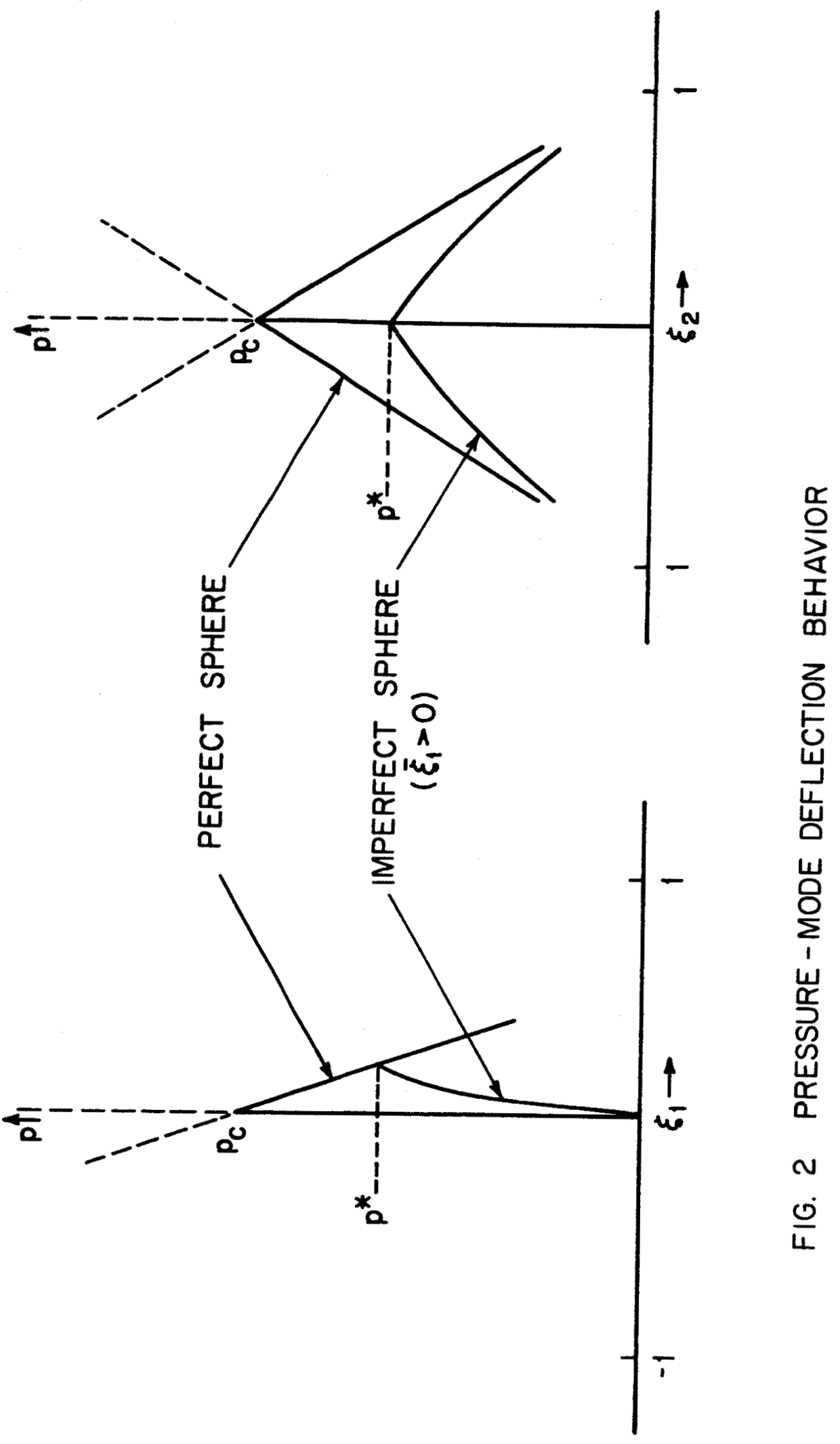




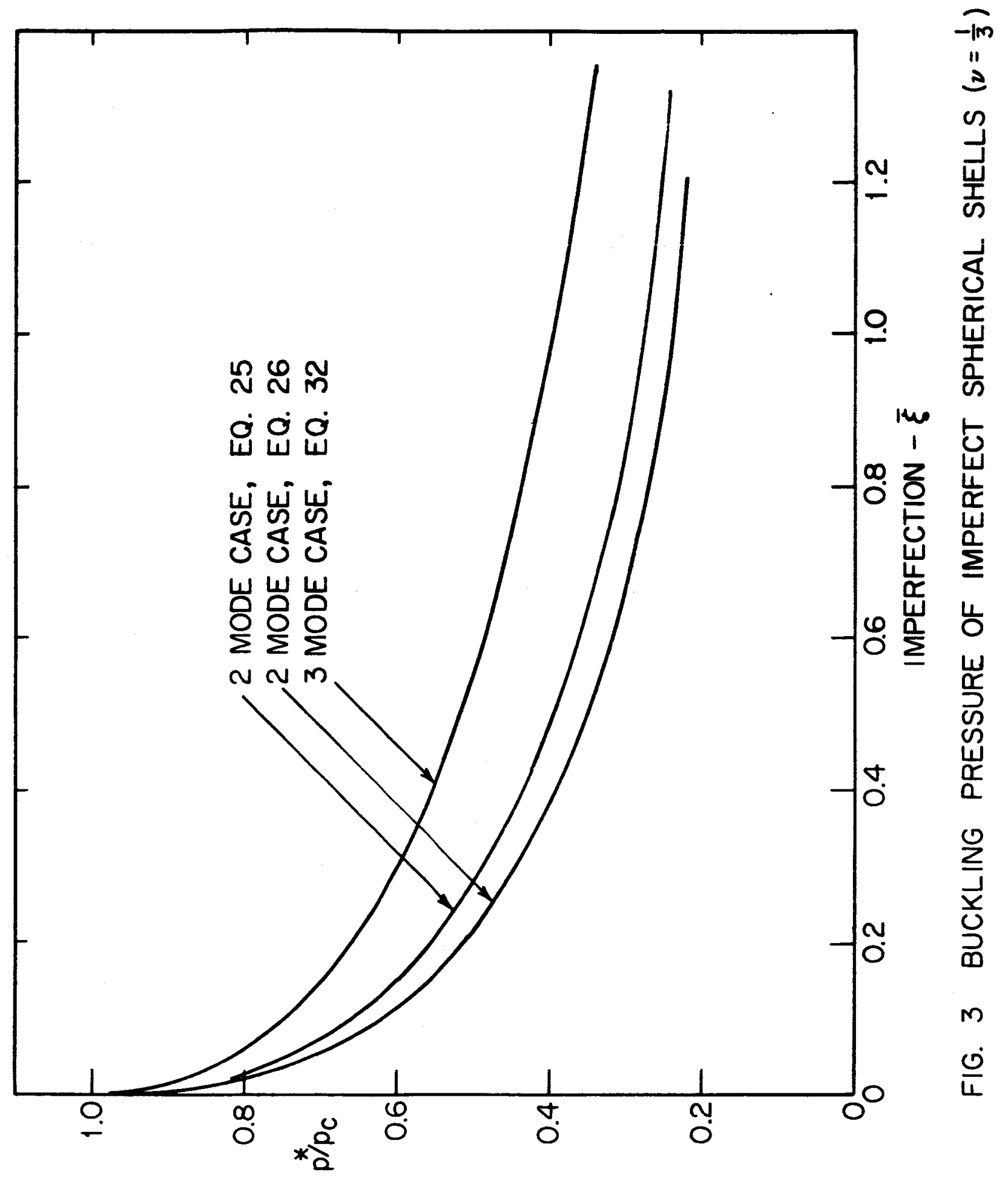




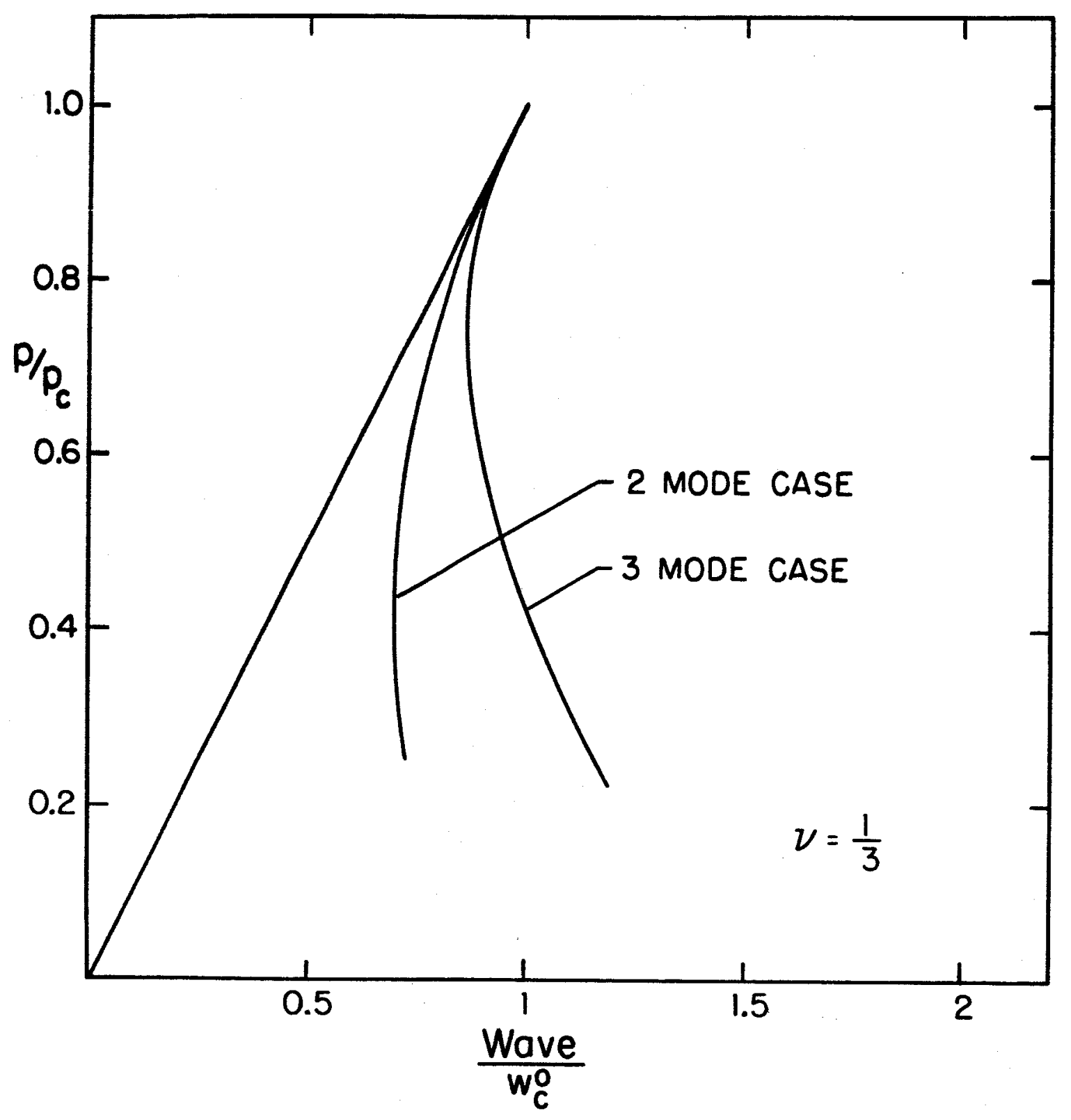

FIG. 4 GENERALIZED LOAD-DEFLECTION CURVES FOR SHALLOW SECTION OF PERFECT SPHERE 


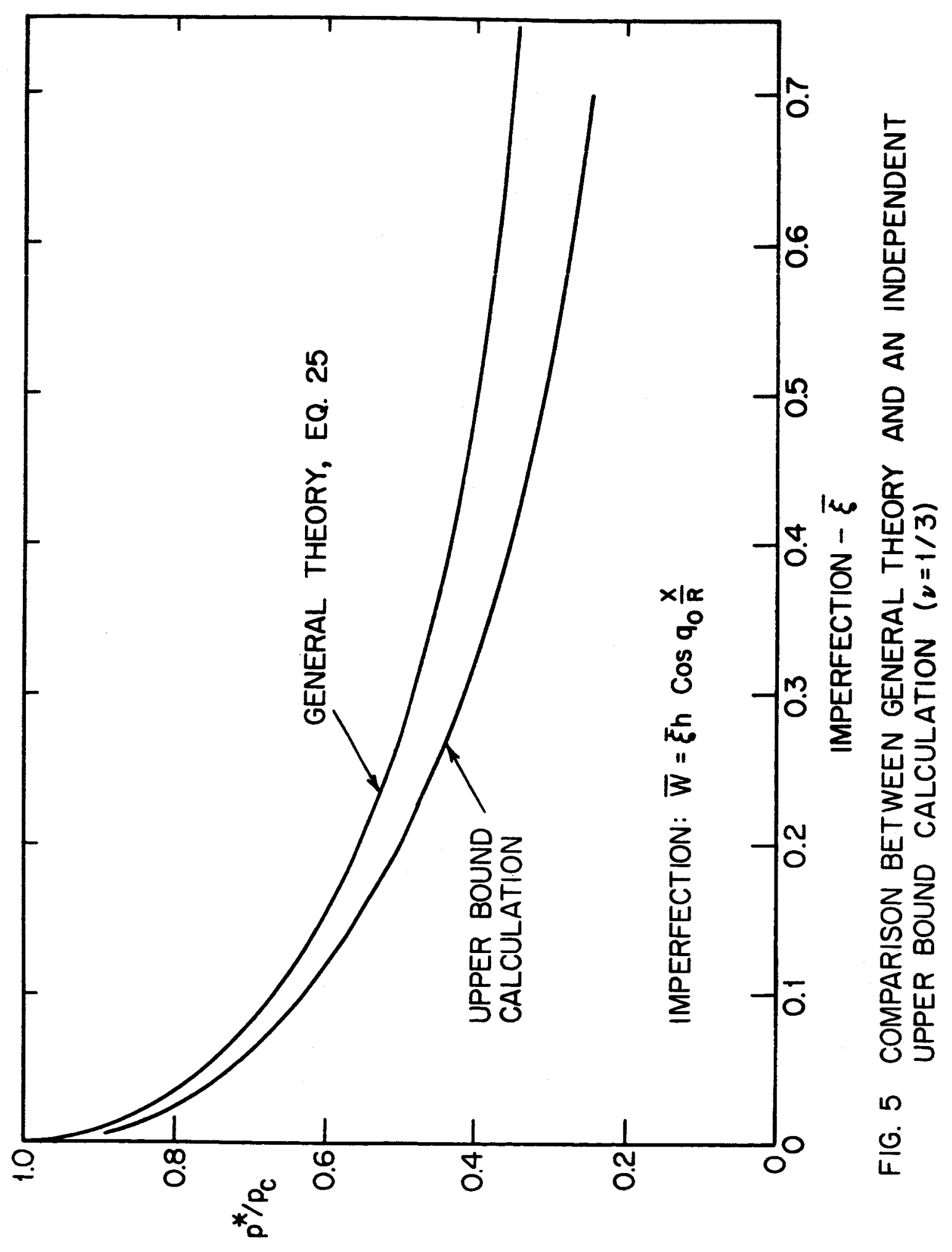

